\title{
Developmental Modulation of Retinal Wave Dynamics: Shedding Light on the GABA Saga
}

\author{
Evelyne Sernagor, ${ }^{1}$ Carol Young, ${ }^{1}$ and Stephen J. Eglen ${ }^{2}$ \\ ${ }^{1}$ School of Neurology, Neurobiology, and Psychiatry, Medical Sciences, University of Newcastle upon Tyne, Newcastle upon Tyne, NE2 4HH, United \\ Kingdom, and ${ }^{2}$ Department of Anatomy and Neurobiology, Washington University School of Medicine, St. Louis, Missouri 63110
}

\begin{abstract}
Embryonic spontaneous activity, in the form of propagating waves, is crucial for refining visual connections. To study what aspects of this correlated activity are instructive, we must first understand how their dynamics change with development and what factors trigger their disappearance after birth. Here we report that in the turtle retina, GABA, rather than glutamate and acetylcholine, influences developmental changes in wave dynamics. Using calcium imaging of the ganglion cell layer, we report how waves switch from fast and broad, when they emerge, to slow and narrow a few days before hatching, coinciding with the emergence of excitatory $\mathrm{GABA}_{\mathrm{A}}$ receptor-mediated activity. Around hatching, waves gradually become stationary patches, whereas $\mathrm{GABA}_{\mathrm{A}}$ shifts from excitatory to inhibitory, coinciding with the upregulation of the cotransporter KCC2, suggesting that changes in intracellular chloride underlie the shift. Dark-rearing from hatching causes correlated spontaneous activity to persist, whereas $\mathrm{GABA}_{\mathrm{A}}$ responses remain excitatory, and $\mathrm{KCC} 2$ expression is weaker. We conclude that GABA plays an important regulatory role during the maturation of retinal neural activity. Using a simple and elegant mechanism, namely the switch from excitatory to inhibitory, $\mathrm{GABA}_{\mathrm{A}}$ receptor-mediated activity is necessary and sufficient to cause retinal waves to stop propagating, ultimately leading to the disappearance of correlated spontaneous activity. Moreover, our results suggest that visual experience modulates the GABAergic switch.
\end{abstract}

Key words: retinal waves; retinal development; GABA; calcium imaging; dark-rearing; visual experience; spontaneous activity

\section{Introduction}

Embryonic retinal ganglion cells (RGCs) fire in spontaneous bursts of action potentials (Sernagor et al., 2001) that are correlated between neighbors (Maffei and Galli-Resta, 1990; Zhou, 1998; Grzywacz and Sernagor, 2000), resulting in waves propagating across the ganglion cell layer (GCL) and the inner nuclear layer (INL) (Meister et al., 1991; Wong et al., 1993, 1995; Feller et al., 1996; Wong et al., 1998; Sernagor et al., 2000, 2001; Zhou and Zhao, 2000). These waves are believed to encode unique spatiotemporal patterns that could guide Hebbian development of visual connections (Katz and Shatz, 1996; Crair, 1999; Eglen, 1999; O’Donovan, 1999; Penn and Shatz, 1999; Wong, 1999).

Pharmacological studies indicate the relative importance of different neurotransmitters in wave propagation. Nicotinic cholinergic neurotransmission is necessary to generate RGCcorrelated spontaneous bursting activity (SBA) (Sernagor and Grzywacz, 1996, 1999) and to propagate waves at early developmental stages (Feller et al., 1996; Catsicas et al., 1998; Wong et al., 1998; Bansal et al., 2000; Sernagor et al., 2000; Wong and Wong, 2000; Zhou and Zhao, 2000). Later, glutamate modulates the

Received Dec. 27, 2002; revised June 24, 2003; accepted July 2, 2003.

This work was funded by the Biotechnology and Biological Sciences Research Council (E.S.) and the Wellcome Trust (S.J.E.). We thank C. Slater for letting us use his equipment for analyzing KCC2 immunofluorescence and J. Payne for his help with selecting the appropriate KCC2 antibodies. We thank J. Demas, C. Slater, and R. Wong for critical reading of this manuscript.

Correspondence should be addressed to Evelyne Sernagor, School of Neurology, Neurobiology, and Psychiatry, Medical Sciences, University of Newcastle upon Tyne, Framlington Place, Newcastle upon Tyne, NE2 4HH, UK. E-mail: Evelyne.Sernagor@nd.ac.uk.

Copyright $\odot 2003$ Society for Neuroscience $\quad 0270-6474 / 03 / 237621-\bullet \$ 15.00 / 0$ waves (Wong et al., 1998; Bansal et al., 2000; Sernagor et al., 2000; Wong and Wong, 2000; Zhou and Zhao, 2000). During the switch, acetylcholine influences the wave size, whereas glutamate modulates their speed (Sernagor et al., 2000). Nicotinic cholinergic neurotransmission also switches to muscarinic receptordriven activity in neonatal rabbit (Zhou and Zhao, 2000). Finally, $\mathrm{GABA}_{\mathrm{A}}$ and glycinergic responses shift from functionally excitatory to inhibitory at P15-P18 in ferret RGCs (Fischer et al., 1998), whereas a prenatal excitatory glycinergic drive becomes inhibitory in rabbit (Zhou, 2001).

Surprisingly little effort has concentrated, however, on longterm developmental changes in wave dynamics, and little is known about the factors causing waves to disappear after birth. Light itself may be important, because SBA disappears shortly after eye opening in mammals (Wong et al., 1993), and it persists in dark-reared turtles (Sernagor and Grzywacz, 1996).

If retinal waves change during development, then the way they instruct the refinement of connections in the visual system could also change (Shatz, 1996). Studies making chronic gross changes to both the amount and the pattern of retinal activity (Penn et al., 1998; Rossi et al., 2001; Stellwagen and Shatz, 2002) show profound changes in the organization of retinal targets. However, to understand how wave dynamics guide the precise carving of visual connections such as retinotopy, the first step is to know how waves change during development and to elucidate the cellular mechanisms underlying these changes.

The goal of this study was to document how the dynamics of turtle retinal waves change with development and to elucidate the 
cellular mechanisms underlying these changes. Because we already know that visual experience is necessary for SBA to disappear in turtle RGCs (Sernagor and Grzywacz, 1996), this model is ideal for investigating how light may modulate the disappearance of spontaneous correlated activity.

Parts of this study have appeared previously (Sernagor and Mehta, 2001; Sernagor et al., 2001) and in abstract form (Sernagor and Eglen, 2001; Sernagor, 2002).

\section{Materials and Methods}

Surgical procedure, dye labeling of the retina, and drug application. Our study was performed on the turtle species Pseudemys scripta elegans. Embryonic ages were determined according to specific staging criteria (Yntema, 1968). S22 corresponds to $\sim 3$ weeks before hatching, S25, to 1 week before hatching, and S26 to the hatching process. Embryos were kept at $29^{\circ} \mathrm{C}$ in a dark incubator. Newly hatched turtles were immediately transferred to water tanks kept at $28^{\circ} \mathrm{C}$, either in $12 \mathrm{hr}$ light/dark cycles (for normal rearing) or in constant darkness. The surgical procedures are described elsewhere (Sernagor and Grzywacz, 1995). RGC retrograde loading with calcium green dextran (CGD) was similar to our method used in the chick (O'Donovan et al., 1993; Sernagor et al., 2000), with the difference that eyecups were incubated overnight in Ringer's solution (Sernagor and Grzywacz, 1995) at room temperature. After isolation, retinas were mounted, GCL facing up, onto gray blotting paper (Millipore, Bedford, MA) and transferred to the experimental chamber onto the stage of an upright microscope (AX70; Olympus Optical, Tokyo, Japan). The chamber was continuously perfused $(2-5 \mathrm{ml} / \mathrm{min})$ with oxygenated Ringer's solution (containing $\mathrm{KCl} 4.9$ instead of $2.9 \mathrm{~mm}$, to increase background spontaneous activity) kept at $26-28^{\circ} \mathrm{C}$.

Pharmacological blockers (Sigma, St. Louis, MO; or Tocris Cookson, Bristol, UK) were bath-applied through the perfusate. GABA, however, was applied with a micropipette in single puffs of $50 \mu \mathrm{l}$ of a $5 \mathrm{~mm}$ solution to avoid receptor desensitization. Because the volume of the bath is $\sim 1$ $\mathrm{ml}$, the final concentration of GABA was $100 \mu \mathrm{M}$. This peak concentration was reached only briefly because the perfusion was kept on during the application of the drug. GABA was always applied near the chamber inflow and then diffused toward the retina placed in the center of the chamber, with the imaged region $\sim 1.2 \mathrm{~cm}$ away from the inflow. As control, puffs of Ringer's solution had no effect on spontaneous activity $(N=3)$, suggesting that merely puffing did not trigger activity by mechanical stimulation. Drug washout led to full activity recovery within 15-30 min.

Analysis of calcium transients. The imaging techniques and analysis of the calcium transients were similar to those used in our previous study in the chick (Sernagor et al., 2000). Briefly, RGCs labeled with CGD were viewed at $20 \times$ (which is sufficient to resolve individual cells) (see Fig. 1) over a field of view of $\sim 600 \times 600 \mu \mathrm{m}^{2}$, which represents approximately a fifth of the retina. Fluorescence changes were detected using a Video Rate Intensified CCD camera (Princeton Scientific Instruments, Monmouth Junction, $\mathrm{NJ}$ ) and recorded continuously (25 frames/sec) onto videotape while simultaneously viewed with the imaging software MetaMorph (Universal Imaging Corporation, Downingtown, PA).

Episodes of activity were digitized (MetaMorph) to measure the average fluorescence over time in many cells, typically 150-200 per retina (Sernagor et al., 2000). Each event was normalized such that the mean of the first five frames of activity was 100\%; we then measured deviations from this baseline. Traces were smoothed using an exponential smoothing function (half-life 3.5 frames). The onset time of a calcium elevation within a trace was the time at which the trace exceeded $20 \%$ of its peak value. To calculate the relative onset plots, a cell that was activated early in a wave was chosen as the reference cell. Then, for every other active cell, we plotted the difference in onset time for that cell and the reference cell as a function of the distance between them. The first-order moments (Sernagor et al., 2000) relative to the wave center, calculated parallel and perpendicular to the dominant orientation, were averaged to estimate wave spatial extent. Wave speed was calculated by dividing the difference in onset times for two cells by the distance between them and averaging over several cell pairs (at least five pairs per wave) (Sernagor et al., 2000).
Immunocytochemical localization of KCC2. Eyes were fixed in 1\% PBS paraformaldehyde for $11 / 2 \mathrm{hr}$ and subsequently washed in $0.1 \mathrm{M}$ PBS. Eyecups were cryoprotected in $30 \%$ sucrose overnight at $4^{\circ} \mathrm{C}$, embedded in a mixture of gelatin and O.C.T. (Sakura, The Netherlands) and frozen in isopentane cooled in liquid nitrogen. Serial cross-sections $(20 \mu \mathrm{M})$ were prepared using a Microm HM560 cryostat, mounted on chromealum-gelatin-subbed slides and stored at $-40^{\circ} \mathrm{C}$. Alternate $10 \mu \mathrm{m}$ sections were collected from the same eyecups for hematoxylin and eosin (H\&E) staining (see below).

We used a standard immunolabeling protocol (Vu et al., 2000). All antibodies were diluted in PBS containing 3\% bovine albumin and $0.1 \mathrm{M}$ L-lysine monohydrochloride. Sections were soaked for $10 \mathrm{~min}$ in $0.1 \%$ Triton X-100 in PBS followed by a brief rinse in PBS. They were incubated overnight at $4^{\circ} \mathrm{C}$ in 1:200 diluted rabbit polyclonal anti-KCC2 primary antibody (Upstate Biotechnology, Lake Placid, NY) raised against a 111 amino acid fusion protein (Payne et al., 1996). After three washes (10 min each) in PBS, slides were incubated for $2 \mathrm{hr}$ at room temperature in tetramethylrhodamine-conjugated swine anti-rabbit IgGs (DakoCytomation, Cambridge, UK), washed in PBS and mounted in Vectashield (Vector Laboratories, Peterborough, UK). Negative controls consisted of alternate sections from the same retinas from which the primary antibody was omitted. Sections were viewed at $40 \times$ using a Leica DMRA fluorescence microscope, and images from sections prepared on the same day were captured at the same exposure $(1 \mathrm{sec})$ with a SPOT digital camera (Diagnostic Instruments Inc., Sterling Heights, MI) using MetaMorph. KCC2 labeling bandwidth [thickness of labeling within the inner plexiform layer (IPL)] and labeling intensity (gray levels) were measured in MetaMorph by drawing rectangles (length = KCC2 bandwidth in IPL; width $=25$ pixels) perpendicular to the IPL. These rectangles were drawn only in places where there was a clear delimitation between labeled and nonlabeled areas (sometimes the labeling is not strictly limited to the IPL, it is often seen on the membrane of cell bodies) (see Fig. $8 A, B$ ). For each section labeled with KCC2 antibodies, we processed an adjacent section with hematoxylin and eosin to measure the entire IPL thickness (see next section). Values in Figure $8 E$ were obtained by normalizing (measured with percentage) all "raw" KCC2 bandwidth measurements to the mean IPL thickness at each corresponding stage. The average labeling intensity (within the rectangles) was normalized (measured with percentage) to the peak value obtained at $\mathrm{PH} 3$ (see Fig. 8 F).

Hematoxylin and eosin staining technique. We used this standard histological technique to measure the IPL thickness. Slides were immersed in Mayer's hematoxylin for 10-15 min, washed under running water, counterstained in eosin for $4-5 \mathrm{~min}$, washed again under running water, dehydrated, cleared, and mounted in Histomount. Sections were viewed under bright-field, and images captured as for KCC2 immunofluorescence. The IPL thickness was then measured in Metamorph.

\section{Results}

\section{Developmental changes in wave dynamics}

We started investigating retinal waves at S22, 3 weeks before hatching (gestation lasts 8 weeks), when turtle RGCs start firing spontaneous correlated bursts of action potentials (Sernagor and Grzywacz, 1995, 1999; Grzywacz and Sernagor, 2000; Sernagor et al., 2001, Sernagor and Mehta, 2001). All our optical recordings were made from central retina, where the density of labeled cells was highest (Fig. 1A). Individual RGCs exhibited strong spontaneous increases in fluorescence, often in oscillations, presumably reflecting recurring bursts of spikes (Fig. $1 B$ ), as we commonly observe with conventional electrophysiological techniques (Sernagor and Grzywacz, 1995, 1999; Grzywacz and Sernagor, 2000; Sernagor et al., 2001) (see also Sernagor et al., 2000).

At S22, waves are relatively fast, propagating at $233.6 \pm 14.7$ $\mu \mathrm{m} / \mathrm{sec}$ (mean $\pm \mathrm{SE})(N=18,6$; first number, waves; second number, retinas) and broad, with a mean moment (an estimate of spatial extent) of $121.7 \pm 3.9 \mu \mathrm{m}(N=18,6)$, recruiting $78.3 \pm$ $4.7 \%$ of RGCs on their trajectory $(N=18,6)$. A propagating wave at S22 is illustrated in Figure 1C. As in other species (Sernagor 
A

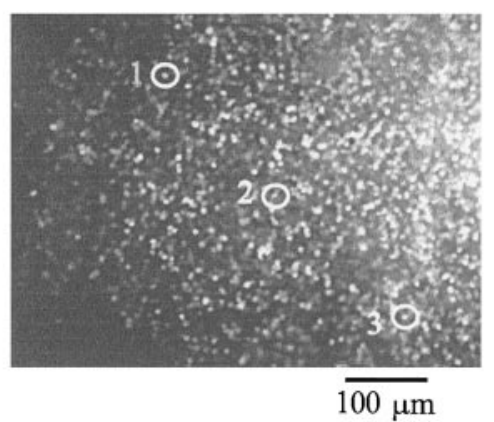

$\mathrm{C}$
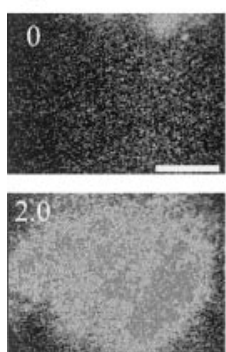

B

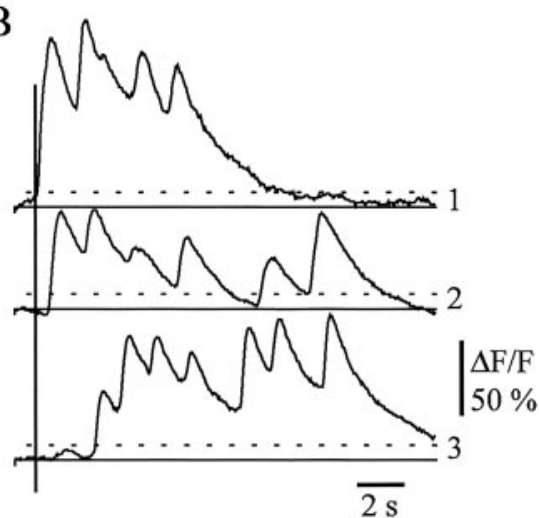

Figure 1. Retinal waves in the turtle embryo. A, Raw image (averaged over 60 frames) of calcium green dextran-labeled $\mathrm{GCL}$ from a whole-mount $\$ 24$ retina. Each dot represents a labeled RGC. The white circles delimit three cells whose activity is illustrated in $B$. $B$, Video rate recording of increases in fluorescence intensity $(\Delta F / F)$ during a spontaneous wave of activity for the three cells demarcated in $A$. The horizontal dotted line shows the threshold for activity onset. The vertical line shows the onset time for cell 1 , the first to become recruited in the wave. Delays to onset increase from cell 1-3, indicating wave propagation. All three cells exhibit recurring outbursts of activity during the wave, indicating the quality of temporal resolution in our recordings. C, Timelapse images of a $\mathrm{Ca}^{2+}$ wave in a S22 retina. The background fluorescence has been subtracted from the images, so that only changes in fluorescence are apparent. Each image illustrates a single video frame obtained at a particular time elapsed from the beginning of the recording (indicated in seconds in the top left side of the image). The wave propagates from top (time 0 ) to bottom. Scale bar, $250 \mu \mathrm{m}$.

et al., 2001), waves in turtle originate at random locations and do not show a preferential direction of propagation. The spatiotemporal characteristics of the waves do not change significantly over the next 10 d, between S23 and S24 (Fig. 2A-C) (see movie 1, available at www.jneurosci.org, for a wave at S23). The waves remain fast $[226.4 \pm 14 \mu \mathrm{m} / \mathrm{sec}(N=36,10)]$ and broad, with a mean spatial extent of $123.1 \pm 2.2 \mu \mathrm{m}(N=41,10)$, recruiting $68.3 \pm 3.2 \%$ of the cells as they sweep through the GCL $(N=43,10)$.

However, by S25, 1 week before hatching, the waves suddenly slow down, becoming narrow and sinuous (see movie 2, available at www.jneurosci.org). To visualize these developmental changes in propagation, Figure 2 (top and middle rows) shows the activity onset of cells relative to a reference cell. The top row shows examples of wave propagation from four different S22 retinas. S22_A to S22_C illustrate a single wave, and S22__ D illustrates two consecutive waves propagating in the same direction. Propagation patterns in these examples are remarkably similar, indicating smooth propagation, without much variability. The middle row shows relative onset plots obtained in four different retinas at S25. Wave propagation is much slower. S25_A, B, and D exhibit complicated, "zigzagging" propagation patterns. A summary of the developmental changes in wave speed, spatial extent, and cellular recruitment is illustrated in Figure $2 A-C$, respectively. All three parameters decrease significantly at S25 (ANOVA, $p<0.0001$ ). Wave speeds undergo the most dramatic decrease, dropping by $85.7 \%(N=19,5)[p<0.001$, NewmanKeuls multiple comparison post-test (N-K test)]. The wave spa-

tial extent and the cellular recruitment decrease, respectively, more modestly by $19.7 \%(N=22,5 ; p<0.001, \mathrm{~N}-\mathrm{K}$ test $)$ and by $37 \%(N=26,5 ; p<0.001, N-K$ test).

One week later, when turtles are about to hatch (S26), once again spontaneous activity patterns change substantially because waves no longer propagate. Instead, patches of coactive neighboring RGCS were observed, which sometimes propagated, but over much smaller areas than before (Fig. $3 A$ ). The top row of Figure 3 illustrates four examples of relative onset plots at S26. The plots reveal sets of nearly horizontal lines, each line representing one patch of coactivated RGCs, with its length reflecting the spread of the patch. Like waves at earlier stages, the patches occur at random locations, and they are not confined to the same RGCs, but are rather dynamic. Once turtles have hatched, the spontaneous patches become smaller and less frequent (Fig. 3, middle panel). Figure $3 B$ shows the steady decrease in cellular recruitment during spontaneous activity from S26 to 3 weeks $\mathrm{PH}$ (PH3), the entire period of patchy activity, indicating a weakening of spontaneous correlated activity in RGCs before it disappears, at approximately $\mathrm{PH} 4$. Cellular recruitment is now arbitrarily calculated over consecutive $5 \mathrm{sec}$ intervals, because periodic, wavelike, activity has been replaced by more continuous activity across the population of cells. Cellular recruitment decreases by $28.7 \%$ between S26 $(N=83$ intervals, 6$)$ and PH1 $(N=60,4)$ $(p<0.001 ; \mathrm{N}-\mathrm{K}$ test). It then drops once again by $21.3 \%$ between PH2 $(N=75,4)$ and PH3 $(N=75,6)(p<0.05, \mathrm{~N}-\mathrm{K}$ test).

\section{Acetylcholine, glutamate, and wave dynamics}

In our search for the factors responsible for the dramatic changes in wave dynamics at S25, we first investigated potential developmental changes in the contribution of acetylcholine and glutamate because both are necessary to generate spontaneous activity in turtle RGCs from early developmental stages (Sernagor and Grzywacz, 1999; Sernagor et al., 2001), and also to propagate retinal waves (Sernagor et al., 2001). Moreover, there is a clear developmental switch in wave control from acetylcholine to glutamate in chick (Wong et al., 1998; Sernagor et al., 2000) and in mammals (Bansal et al., 2000; Wong and Wong, 2000; Zhou and Zhao, 2000). We observed the same effects of partial cholinergic and glutamatergic receptor blockade (with low doses of antagonists, because high doses completely block SBA) on wave dynamics as previously found in the chick (Sernagor et al., 2000) (Fig. 4). However, unlike in chick and mammals, both neurotransmitters were required at all embryonic stages tested (S23 to S25). During partial nicotinic cholinergic receptor blockade (with mecamylamine $0.5-1 \mu \mathrm{M})$, the waves shrank: their spatial extent and cellular recruitment decreased, respectively, by $45.3 \%(N=$ $33,4$; $p<0.0001$, two-tailed $t$ test $)$ and by $58.8 \%(N=32,4 ; p<$ 0.0001 , two-tailed $t$ test), whereas the speed did not change 
$(N=25,4 ; p=0.1719)$. During partial glutamatergic blockade (with $\gamma$-GLU-GLY, a broad spectrum antagonist or Chicago Sky Blue, a glutamate uptake inhibitor, 2-5 $\mu \mathrm{M}$ ), the wave speed decreased by $30.9 \%(N=62$, $7 ; p<0.0067$, two-tailed $t$ test), whereas the spatial extent $(N=62,7 ; p=0.2645)$ and cellular recruitment $(N=61,7 ; p=0.7091)$ did not change.

\section{Developmental changes in}

$\mathrm{GABA}_{\mathrm{A}}$ responses

Given the importance of $\mathrm{GABA}_{\mathrm{A}}$-mediated activity in other aspects of developing circuits (Leinekugel et al., 1999; Ben-Ari, 2002), we chose to test its role in generating correlated spontaneous activity. First we blocked endogenous GABA with the $\mathrm{GABA}_{\mathrm{A}}$ antagonist bicuculline $(1-10 \mu \mathrm{M})$ at various developmental stages. Between S22 and S24, bicuculline had no noticeable effect on wave dynamics (nine retinas). At S25, however, when waves suddenly become slower and more winding, bicuculline became effective at modulating the waves. At low concentrations (1-5 $\mu \mathrm{M})$, the drug abolished the waves (four retinas). At higher concentrations, however, bicuculline did not block the waves, but on the contrary, it accelerated them (Fig. 5A). Wave speed dramatically increased by $886.5 \%$ in the presence of $20 \mu \mathrm{M}$ bicuculline $(N=16,2 ; p<0.0003$, two-tailed $t$ test), up to $211.5 \pm 39.5 \mu \mathrm{m} / \mathrm{sec}$, similar to wave speed in younger embryos (see above). In contrast, there was no effect on spatial extent $(N=16,2 ; p<0.7680$, twotailed $t$ test) and cellular recruitment $(N=$ 16,$2 ; p<0.2969$, two-tailed $t$ test).

To clarify the intriguing effect of GABA on retinal activity at S25, we applied single puffs of GABA in the experimental chamber. The purpose of this was to test whether GABA would produce excitatory or inhibitory responses in RGCs. Single puffs indeed could induce elevated calcium levels in single cells. However, much to our surprise, GABA puffs triggered very large waves of excitation (Fig. 6, movie 3, available at www.jneurosci.org). The dynamics of these "tidal" waves of excitation were completely different from those of spontaneous waves. They propagated slowly, at $52.3 \pm 1.8 \mu \mathrm{m} / \mathrm{sec}(N=4$, $4)$, recruiting all RGCs, and they were long lasting (38.2 $\pm 5.5 \mathrm{sec}$ ) (Fig. 6B), especially when compared with the spontaneous waves observed at S25 (Fig. 2A,C). Given the great difference in dynamics between these GABA-induced waves and spontaneously generated waves, we suggest that different mechanisms are involved in the propagation of these two types of waves (see Discussion).

As we have reported above, waves stop
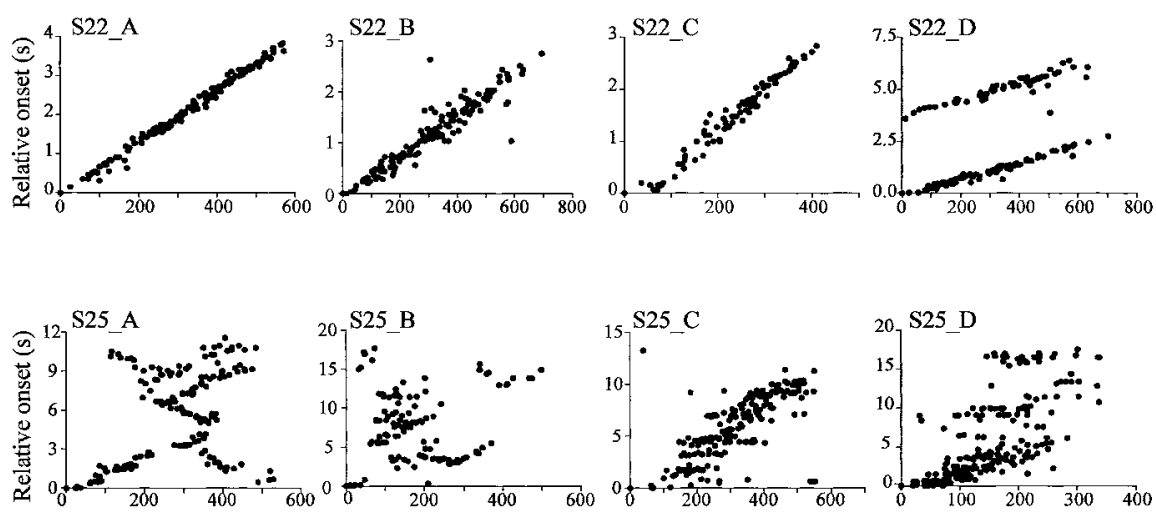

Distance $(\mu \mathrm{m})$
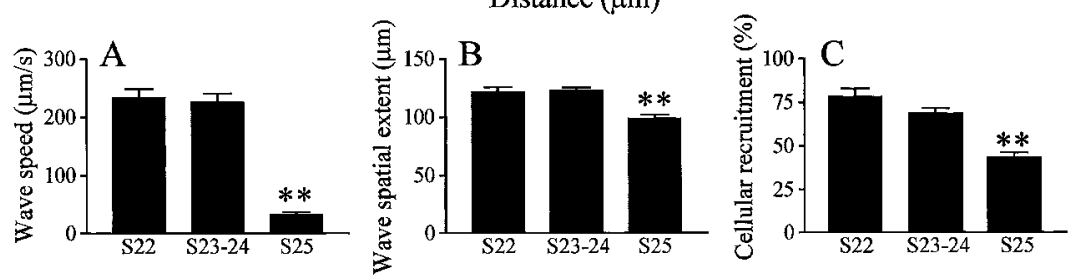

Figure 2. Developmental changes in wave dynamics. $S 22 \quad A-S 22 \quad$ D, Relative onset plots from four different $\$ 22$ retinas. S22 D D shows two consecutive waves ( 3 sec apart) propagating in the same direction, as indicated by the similar slopes. Waves are fast and have a uniform front, as indicated by a linear increase in delays to onset with distance from the reference cell. S25_A $A-S 25 \quad D$, Relative onset plots from four different S25 retinas. The plots reveal that waves are much slower and more winding than at S22. A-C respectively illustrate developmental changes (between S22 and S25) in wave speed, spatial extent, and cellular recruitment within waves. All three parameters decrease significantly at $\$ 25$. Asterisks indicate statistical significance between that bar and the previous one ${ }^{* *} p<0.001$; N-K post test). Error bars indicate SEM.
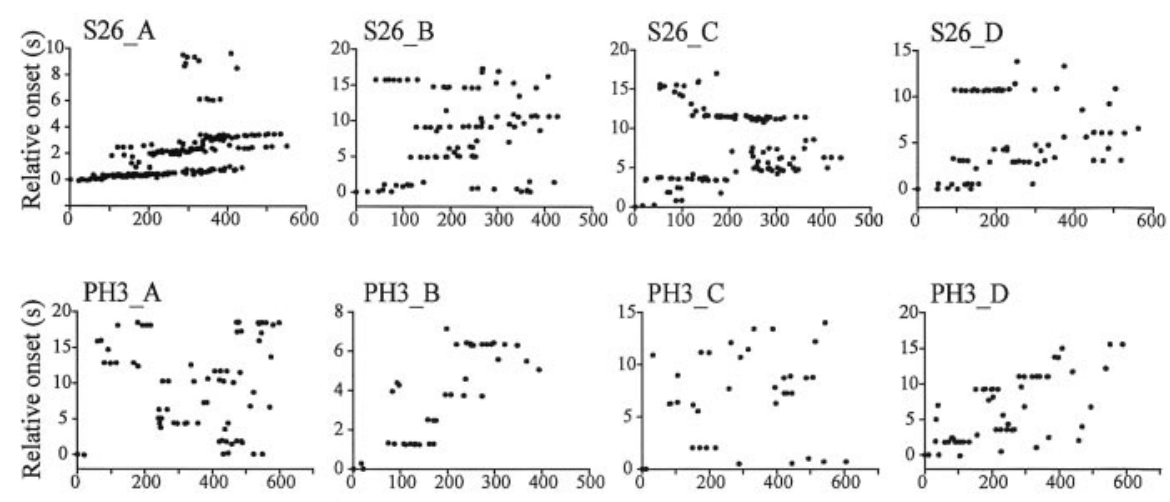

Distance $(\mu \mathrm{m})$
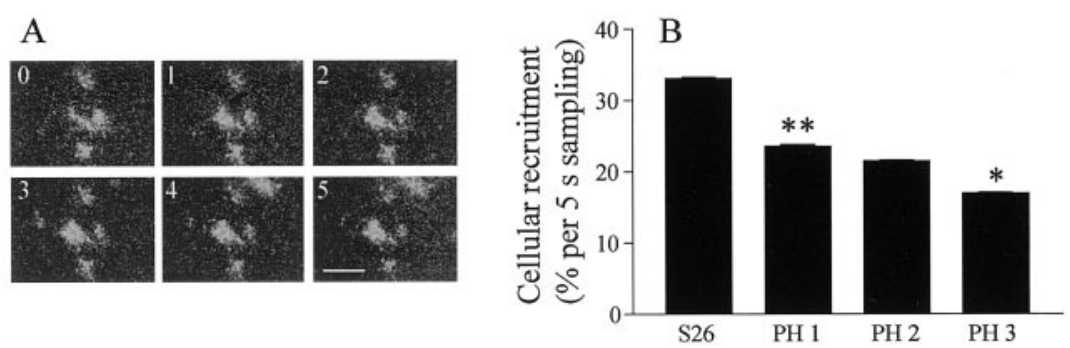

Figure 3. Waves become localized activity patches at hatching. $S 26 \_A-S 26 \_$D, Relative onset plots from four different retinas at $\mathrm{S} 26$. The plots reveal patches of synchronized activity across $\mathrm{RGCs}$. In $\mathrm{S} 26$ _ A, there is still some propagation because the lines are slightly oblique. $\mathrm{PH} 3 \_$_ $A-P H 3 \quad$ D , Relative onset plots from four different retinas at 3 weeks PH. Patches are now smaller and recruit fewer cells. $A$, Time-lapse images of spontaneous activity in a $\$ 26$ retina. Conventions are like Figure $1 C$. The activity is now restricted to local patches. $B$, Decrease in cellular recruitment during spontaneous activity from S26 onward. Asterisks indicate statistical significance between that bar and the previous one ( ${ }^{* *} p<0.001 ;{ }^{*} p<0.05 ; \mathrm{N}-\mathrm{K}$ post-test). Error bars indicate SEM. 


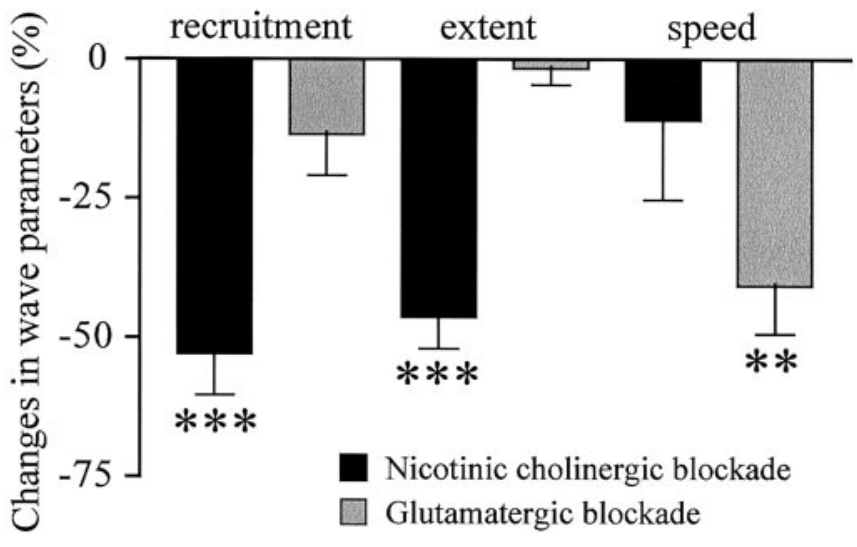

Figure 4. Effects of partial cholinergic and glutamatergic blockade on wave dynamics. Percentage difference from control in cellular recruitment, wave spatial extent, and speed in the presence of cholinergic nicotinic (black bars) or glutamatergic (gray bars) antagonists (see Results for more details). Waves shrink during nicotinic blockade, whereas the main effect of glutamate blockade is to slow waves down. Asterisks indicate statistical significance between control and drug $\left({ }^{* * *} p<0.0001 ;{ }^{* *} p<0.0067\right.$; two-tailed $t$ test). Error bars indicate SEM.

propagating around hatching, at S26. Our findings about GABAergic effects at S25 suggest these changes in wave dynamics at S26 might reflect a change in the nature, or polarity of $\mathrm{GABA}_{\mathrm{A}}$ responses, a possibility that was confirmed with bicuculline at S26 and at post-hatching stages. Figure $5 B$ illustrates a doseresponse effect of bicuculline in a S26 retina that had extremely patchy activity in control conditions. At $10 \mu \mathrm{M}$, the drug caused the patches to become much larger, and when the concentration was doubled, at $20 \mu \mathrm{M}$, they reverted to fast propagating waves (218.7 $\pm 41.2 \mu \mathrm{m} / \mathrm{sec}$, calculated over four waves). At lower concentrations $(1-5 \mu \mathrm{M})$, bicuculline increased cellular recruitment in all retinas (Fig. 5C). This enhancing effect increased with development (Fig. 5D). At the same time, GABA-evoked waves were already shorter at S26 than at S25 $(20.9 \pm 3 \mathrm{sec} ; N=4,4)$ (Fig. 6B,C). They were also harder to evoke $\mathrm{PH}$ : they were elicited in four of five retinas at $\mathrm{PH} 1-2$, and only in two of six retinas at $\mathrm{PH} 3$, suggesting that during development, $\mathrm{GABA}_{\mathrm{A}}$ responses slowly shift from being excitatory to inhibitory.

\section{Visual experience, $\mathrm{GABA}_{\mathrm{A}}$, and spontaneous activity}

In all species, RGC-correlated spontaneous activity disappears within the first postnatal month (Sernagor et al., 2001). Although visual experience does not appear to influence the disappearance of retinal waves in mice (Demas et al., 2003), light deprivation in turtles (which become sensitive to light earlier than mammals) (Sernagor and Grzywacz, 1995) causes spontaneous bursting activity to persist, and consequently, receptive fields to become much larger than normal (Sernagor and Grzywacz, 1996; Sernagor and Mehta, 2001). Calcium imaging confirmed the findings that retinas from dark-reared (DR) turtles are considerably more spontaneously active than normal (Fig. 7, top row, compare with $\mathrm{PH} 3$ plots in Fig. 3, second row; movie 4, available at www.jneurosci.org). Both patches (DR4_B, the number indicates weeks of dark-rearing) and propagation (DR3_B, showing two waves propagating in different directions) are frequently seen, and cellular recruitment is much higher than normal at that age (Fig. 7A). Because our observations from normal development suggest that GABA may be shifting polarity during that period (see Results, "Developmental changes in GABA A $_{\mathrm{A}}$ responses"), perhaps causing the disappearance of spontaneous activity, we have investigated GABA responses after DR2-4. To our surprise, we found that GABA retained a substantial excitatory component, even after 4 weeks of dark-rearing. Indeed, GABA-evoked waves were observed in all twelve DR2-4 retinas examined, including six retinas from DR4 turtles (Fig. 7B). These waves were of similar strength, as indicated by cellular recruitment and duration, as in younger $\mathrm{PH}$ retinas from normally reared animals. Although bicuculline $(1-5 \mu \mathrm{M})$ increased cellular recruitment during correlated spontaneous activity in DR retinas by $49.5 \%$ $(N=145,4 ; p<0.0001$, two-tailed $t$ test) (Fig. $7 C)$, the increase was much weaker than in the oldest control animals tested (122.2\% increase) (Fig. 7D), suggesting that as a result of darkrearing, GABA remained excitatory.

\section{Developmental changes in KCC2 expression}

Many studies report that the excitatory effect of GABA in the immature nervous system is caused by elevated intracellular $\mathrm{Cl}^{-}$ concentration $\left(\left[\mathrm{Cl}^{-}\right]_{\mathrm{i}}\right)$ because of the late developmental upregulation (Payne et al., 1996; Rivera et al., 1999; Vu et al., 2000) of the extruding neural $\mathrm{K}^{+} / \mathrm{Cl}^{-}$cotransporter KCC2 (Williams et al., 1999; Delpire, 2000). Using immunofluorescence, we have looked at the expression of KCC2 between S25 and PH3 and at DR3 (Fig. 8). KCC2 was expressed in the IPL and outer plexiform layers (OPL) (Fig. 8) at all stages, but it increased with development in the IPL (we did not study the OPL). To ensure that changes were not simply caused by retinal growth, we normalized our measurements to the IPL thickness (retinal layers were revealed using the $\mathrm{H} \& \mathrm{E}$ stain) (Fig. $8 A-C$ ). IPL thickness increased by $27 \%$ between $S 25$ ( $N=93$ sampling lines, from three retinas) and S26 $(N=93,3)$ (Fig. $8 D)$. The fraction of the IPL labeled with KCC2 (Fig. $8 E$ ) increased from $62.3 \%$ at $\mathrm{S} 25(N=162,3)$ to $93.8 \%$ at $\mathrm{S} 26(N=126,3)$ and $94.3 \%$ at $\mathrm{PH} 3(N=172,4)$ (Fig. $8 A, B)$ (despite a small but significant reduction in the IPL thickness between S26 and PH3) (Fig. 8D), indicating that KCC2 is indeed upregulated with development. The IPL was thicker at DR3 $(21.7 \pm 0.5 \mu \mathrm{m} ; N=117,3)$ than at PH3 (17.9 $\pm 0.4 \mu \mathrm{m}$; $N=83,3$ ) (Fig. $8 D$ ). This is consistent with our previous observations that dark-rearing causes RGC dendritic trees (Sernagor and Mehta, 2001) and receptive fields (Sernagor and Grzywacz, 1996) to grow far beyond normal. Nevertheless, the KCC2 IPL fraction labeling (Fig. $8 \mathrm{E}$ ) was significantly lower than in matching controls, decreasing by $23.5 \%(N=126,3)$. Interestingly, we found that the labeling intensity decreased from S25 to S26, but then increased again and peaked at PH3. These changes are perhaps caused by the initial thickening of the IPL, causing a "dilution" of the labeling, followed by the delayed addition of KCC2 molecules to these new processes. The labeling intensity decreased to its minimum value in DR3 retinas $(12.4 \%$ decrease; $N=126,3)($ Fig. $8 F)$.

\section{Discussion}

In this study, we have reported on the developmental modulation of retinal waves in turtle retina. In brief, waves propagate for approximately 2 weeks, before slowing down and narrowing at S25. Shortly after, the activity weakens until disappearing at approximately PH4. Spatial extent is controlled by acetylcholine, whereas glutamatergic connections influence wave speed. Neither transmitter, however, is responsible for the changes in wave propagation at S25. Instead, GABA, which is excitatory at S25, controls wave dynamics. As GABA becomes inhibitory, stationary patches gradually replace waves. Finally, our dark-rearing studies suggest that light deprivation suppresses the developmental upregulation of $\mathrm{KCC} 2$, causing $\mathrm{GABA}_{\mathrm{A}}$ responses to remain excitatory, and hence maintain waves. 
Our results suggest that spatiotemporal activity patterns change with development, and this has potentially important implications for guiding the establishment of vertebrate visual connections (Wong et al., 1993; Katz and Shatz, 1996; Shatz, 1996; Crair, 1999; Wong, 1999). By interfering with specific propagation aspects at different developmental stages, it will be possible to establish to what extent the information encoded in the waves refines the formation of visual connections. For example, it will be interesting to see how reverting patches to propagating waves influences the size and shape of receptive fields and dendritic arbors in RGCs or their axonal projections as well as tuning of retinotopy in retinal projections.

Our findings suggest that rather than acetylcholine or glutamate, GABA is the major neurotransmitter involved in developmental changes of propagation patterns in turtle. The introduction of excitatory $\mathrm{GABA}_{\mathrm{A}}$ responses at $\mathrm{S} 25$ causes waves to suddenly slow down and then, as GABA becomes inhibitory, waves first become stationary patches and then disappear. Although previous studies in mammals (Bansal et al., 2000; Zhou and Zhao, 2000; Wong and Wong, 2000) and chick (Wong et al., 1998; Sernagor et al., 2000) report a switch from acetylcholine to glutamate, none of them demonstrate that the switch underlies changes in propagation patterns. Moreover, GABA shifts from excitatory to inhibitory at approximately P15-P18 in ferret (Fischer et al., 1998), shortly before waves stop propagating (Wong et al., 1993). A recent study on rabbit has reported similar findings to ours, namely that blockade of $\mathrm{GABA}_{\mathrm{A}}$ (and glycinergic) receptors transforms late, weak, and local-

ized waves to strong propagating ones (Zhou et al., 2002). It is therefore unlikely that the novel role of GABA reported here is unique to reptiles. Estimates from cat retina suggest that at least $30-40 \%$ of amacrine cells are GABAergic (Vaney, 1990), and hence many types of amacrine cells may contribute to the generation and propagation of spontaneous activity. Glycine does not play a major role in spontaneous burst modulation in turtle RGCs (Sernagor and Grzywacz, 1999), and therefore is unlikely to control wave dynamics.

Early excitatory expression of GABA (Leinekugel et al., 1999; Ben-Ari, 2002) and glycine (Kandler and Friauf, 1995; Singer et al., 1998; Ehrlich et al., 1999) is common in the developing CNS. Our findings suggest a strong correlation between the developmental shift in $\mathrm{GABA}_{\mathrm{A}}$ responses and the upregulation of KCC2 (Vu et al., 2000), the neural $\mathrm{K}^{+} / \mathrm{Cl}^{-}$cotransporter responsible for extruding $\mathrm{Cl}^{-}$from the cytoplasm in adult neurons. Remarkably, visual experience may influence this process. Indeed, we find that although the IPL thickness increases at S26, presumably because new dendrites are added, the proportion of IPL dendrites expressing KCC2 also increases. Growth from S25 to S26-PH3 enhances KCC2 expression mainly in the inner part of the IPL,

\section{Control}

Bicuculline $20 \mu \mathrm{M}$

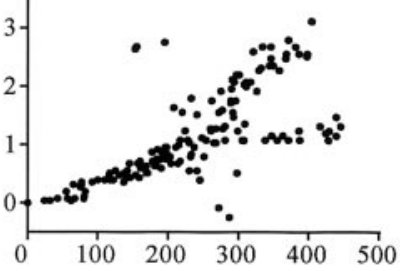

Distance $(\mu \mathrm{m})$

Control
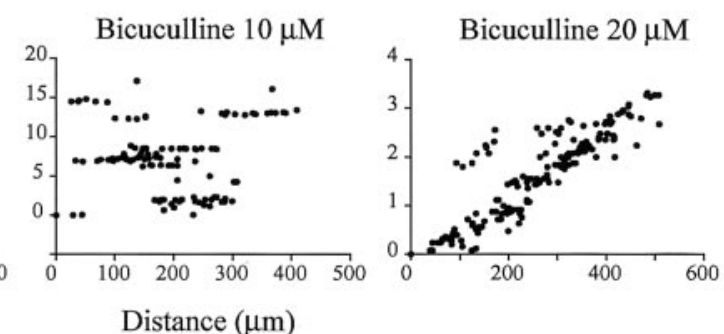

Distance $(\mu \mathrm{m})$

$\mathrm{D}$
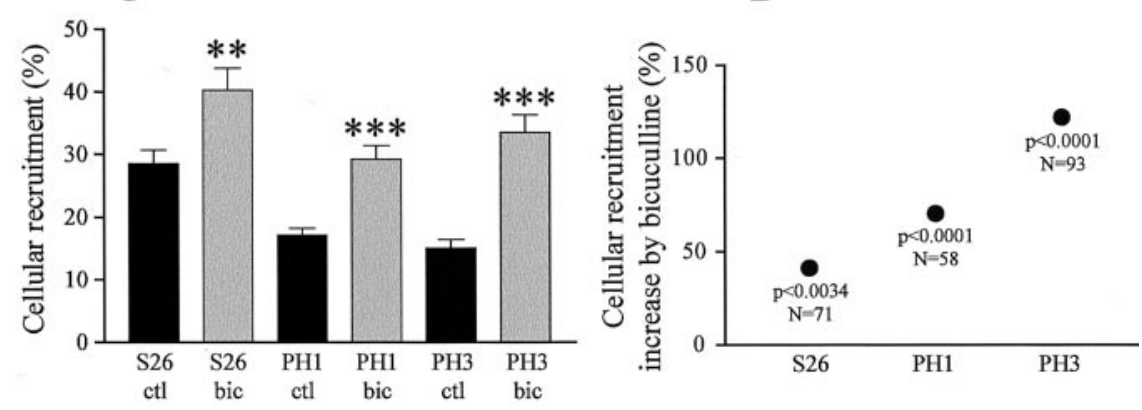

Figure 5. Effects of $G A B A_{A}$ receptor blockade on spontaneous activity. $A$, Relative onset plots from a $S 25$ retina in control conditions and in the presence of bicuculline $(20 \mu \mathrm{M})$. Propagation speed is $\sim 10$-fold faster in bicuculline. B, Relative onset plots from a 26 retina in control conditions, 10 , and $20 \mu \mathrm{m}$ bicuculline. The dose-response effect of bicuculline is remarkable; at $10 \mu \mathrm{m}$ induced increase in cellular recruitment during spontaneous activity from S26 to PH3. Black bars, control; gray bars, bicuculline illustrated in C. The significance of the $t$ test and the number of observations is indicated below each data point. The enhancing effect of bicuculline increases with development, suggesting that GABA becomes inhibitory.

near the GCL, whereas dark-rearing has the opposite effect, suggesting that these changes in KCC2 levels originate from RGC dendrites, where GABAergic amacrine cells contact RGCs. In support of this idea, KCC2 is expressed primarily on RGC dendrites ( $\mathrm{Vu}$ et al., 2000). Furthermore, control PH3 (and S25) retinas exhibit more fluorescent labeling in the inner nuclear layer than DR3 retinas (Fig. 8, compare $B, C$ ). Ultrastructural studies will help us find the sites where KCC2 expression changes. All of this supports our physiological observation that waves stop propagating at approximately S25 to S26 because GABA starts its inhibitory switch. Our results do not exclude the possibility that there is a concomitant developmental downregulation of the $\mathrm{Na}^{+}-\mathrm{K}^{+}-2 \mathrm{Cl}^{-}$cotransporter $\mathrm{NKCC} 1$, known to maintain high $\left[\mathrm{Cl}^{-}\right]_{\mathrm{i}}$ in immature neurons (Delpire, 2000).

One could argue that introducing a new excitatory component to the network at S25 should accelerate and enhance the waves. However, by shunting the membrane to approximately $-40 \mathrm{mV}$, the presumed $\mathrm{Cl}^{-}$reversal potential $\left(\mathrm{E}_{\mathrm{Cl}}\right), \mathrm{GABA}$ cannot evoke spikes, and therefore is not truly excitatory, although it generates "tidal" calcium waves. Based on a perfusion rate of $2-5$ $\mathrm{ml} / \mathrm{min}$ (see Materials and Methods), we estimate that it took 
A
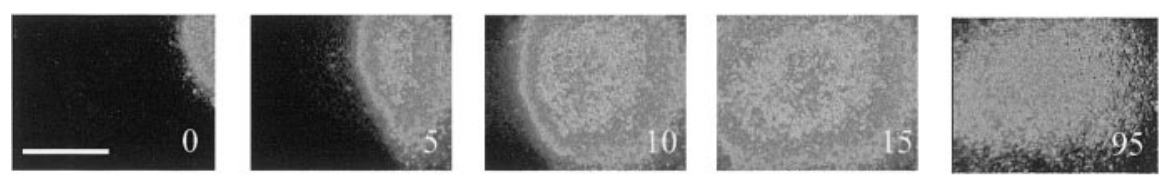

B

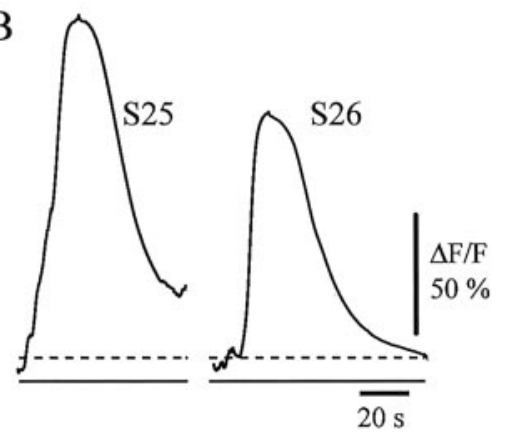

C

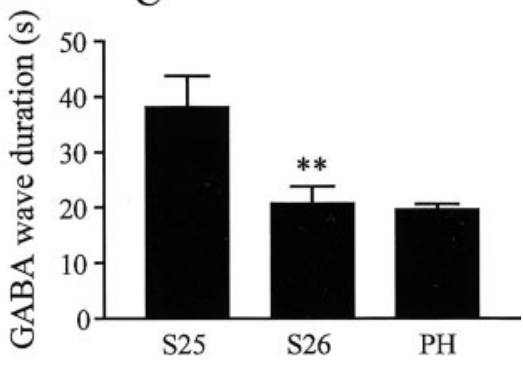

Figure 6. GABA is excitatory at $\$ 25$. A, Time-lapse images of a GABA-induced wave in a S25 retina. Conventions are the same as in Figures 1C and 3A. These "tidal" excitation waves recruit all RGCs, and they are very prolonged (last frame is taken at $95 \mathrm{sec}$ from the beginning). B, GABA-evoked waves at $\$ 25$ and $\$ 26$. Each trace illustrates the activity averaged over the entire population of RGCs analyzed. Individual traces were shifted in time so that all peaks occurred at the same time. Dotted line as in Figure $1 B$. $C$, Decrease in GABA-evoked wave duration from S26 onwards. ${ }^{* *} p<0.001, \mathrm{~N}-\mathrm{K}$ post-test. Error bars indicate SEM.

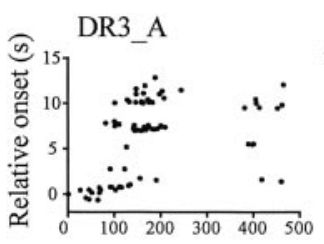

DR3_B

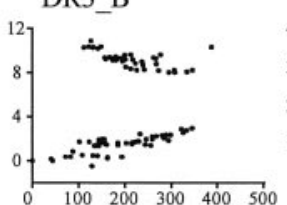

DR4_A

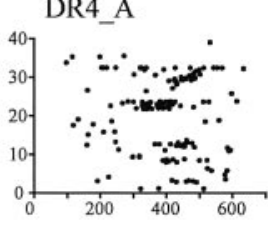

DR4_B

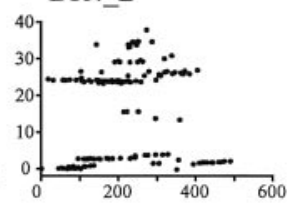

Distance $(\mu \mathrm{m})$
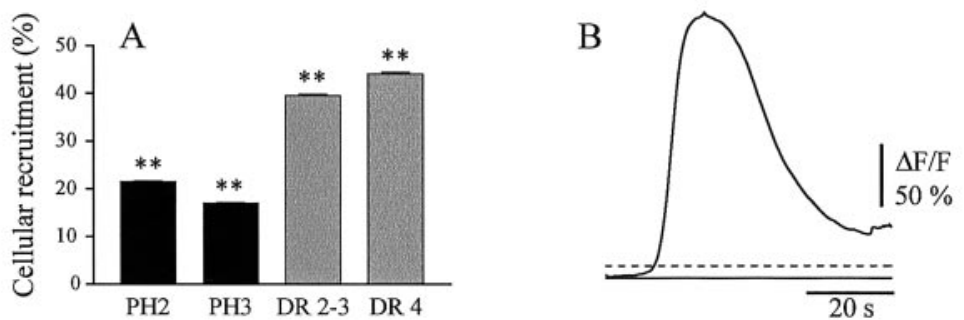

$\mathrm{C}$

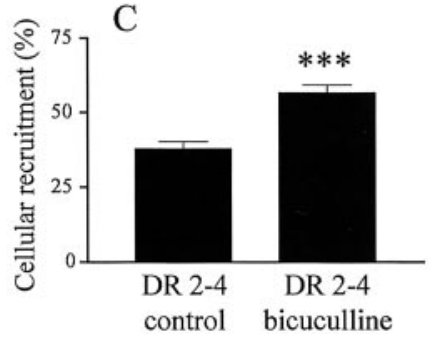

D

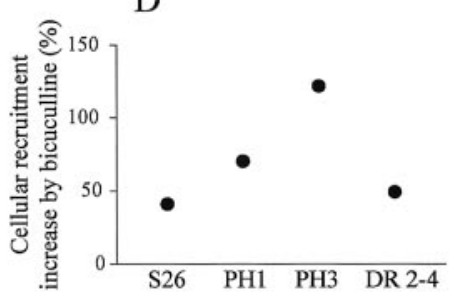

Figure 7. Dark-rearing enhances spontaneous activity and causes GABA to retain an excitatory component. DR3_A-DR4_B, Relative onset plots from two retinas at DR3 and two retinas at DR4. Spontaneous activity is strong (Fig. 3, compare PH3_A to PH3_D), and sometimes propagates smoothly (DR3_B). A, Cellular recruitment at PH2, PH3 (black bars), DR2-3, and DR4 (gray bars). Recruitment is much higher in DR retinas. ${ }^{* *} p<0.001$ (N-K post test, $\mathrm{PH} 2$ compared with DR2-3 and PH3, with DR4). B, GABA-induced wave in a DR4 retina (conventions as in Fig. $6 B$ ). In normal rearing conditions, GABA is already inhibitory at that age. C, Bicuculline-induced $(2-5 \mu \mathrm{m})$ increase in cellular recruitment in DR2-4 retinas. ${ }^{* * *} p<0.0001$. Error bars indicate SEM. $D$, Percentage increase in cellular recruitment induced by bicuculline $(2-5 \mu \mathrm{m})$ from S26 to PH3 and in DR2 -4 . The progressive developmental increase in bicuculline efficacy at enhancing spontaneous activity is much weaker in DR conditions, suggesting that GABA has remained excitatory.

GABA 5-15 sec to reach the retina. GABAevoked waves took longer to appear, $10-30 \mathrm{sec}$ after the puff. Although we cannot refute the possibility that these waves simply result from the diffusion of GABA across the retina, such a delay in wave appearance suggests they are triggered by an intrinsic induction mechanism. In support, waves did not always propagate in the direction of the perfusion flow, but sometimes came from the opposite direction. Occasionally, waves coming from opposite directions even invaded the imaged region simultaneously. Moreover, GABA puffs at older stages did not elicit excitation waves. It is conceivable that these GABA-evoked waves reflect $\mathrm{Ca}^{2+}$ activity rather than spiking, because voltage-gated $\mathrm{Ca}^{2+}$ channels open with depolarization. This could explain the fundamentally different dynamics between GABA-evoked waves and spontaneous waves. Perhaps the prolonged depolarization induced by GABA and $\mathrm{Ca}^{2+}$ influx causes $\mathrm{K}^{+}$efflux, which in turn depolarizes neighboring cells, resulting in these slow but massive and rather uniform waves of excitation. In support of GABA not being truly excitatory (in the sense that it cannot generate sustained spiking activity) although depolarizing at S25, spontaneous wave speed decreases significantly and waves revert to fast propagation in the presence of high concentrations of bicuculline. Moreover, waves are much narrower, recruiting fewer RGCs than at earlier developmental stages. Whether waves, albeit slower and narrower, still propagate at S25 may depend of the fine balance between the respective contributions of acetylcholine, glutamate, and GABA to activity generation and propagation.

The intriguing blockade effect of bicuculline at lower concentrations may be attributable to the removal of an excitatory component provided by $\mathrm{GABA}_{\mathrm{A}}$ responses at this stage. Whether bicuculline produces an inhibitory or an excitatory effect at S25 perhaps depends on the fine balance between the overall membrane depolarization and shunting (to approximately $-40 \mathrm{mV}$ ) caused by GABA. There are, however, other potential explanations to this bimodal effect of bicuculline at S25. One possibility is that at that S25, bicuculline acts on separate subclasses of $\mathrm{GABA}_{\mathrm{A}}$ receptors at low and high concentrations. For example, extrasynaptic $\mathrm{GABA}_{\mathrm{A}}$ receptors might be blocked at lower concentrations than synaptic receptors (Mody, 2001). Another possibility is that $\left[\mathrm{Cl}^{-}\right]_{\text {in }}$ differs among retinal neuronal types because of differential expression of KCC2 at 
S25 (but not at later stages, because later on, activity monotonically increases with drug concentration) (Fig. 5), resulting in different effects of GABA on RGCs and amacrine cells (Vardi et al., 2000). We suggest that by gradually shifting to more hyperpolarized levels, $\mathrm{E}_{\mathrm{Cl}}$ causes the developmental changes in wave dynamics. In support, activity enhancement by bicuculline increases with development, and GABA-evoked waves become shorter and ultimately disappear. More detailed biophysical studies using small, controlled puffs of GABA directly onto synaptically isolated individual RGCs while recording GABA-evoked currents will help understanding of these processes.

Light controlling the polarity of $\mathrm{GABA}_{\mathrm{A}}$ responses is perhaps a new concept in visual development, but not in circadian physiology, where GABA is a major player (Cardinali and Golombek, 1998), and retinal GABA levels are under circadian clock control (Jaliffa et al., 2001). Remarkably, in the suprachiasmatic nucleus, the circadian rhythm generator, $\mathrm{GABA}_{\mathrm{A}}$ responses switch polarity between day and night, presumably because of oscillations in $[\mathrm{Cl}]_{\text {in }}$ (De Jeu and Pennartz, 2002). Retinal GABA levels increase with light activation (Roberts, 1995), and GABA acts as a self-limiting factor for its polarity switch during development (Ganguly et al., 2001). Therefore, perhaps visual experience increases GABA levels in the retina, thereby promoting the switch.

However, light cannot entirely account for the shift because the effect of $\mathrm{GABA}_{\mathrm{A}}$ receptor activation has already started shifting polarity at hatching. Perhaps high levels of GABA itself initially trigger the switch (Ganguly et al., 2001). In turtle, GABA-expressing cells are present long before hatching (Nguyen and Grzywacz, 2000), and recent unpublished observations from our laboratory confirm that chronic $\mathrm{GABA}_{\mathrm{A}}$ activity is necessary even from before hatching to operate the switch.

A recent study in the mouse retina found that dark rearing does not prolong the period during which RGCs exhibit correlated activity (Demas et al., 2003). In control mice, correlated SBA is still present at approximately P15, just after eye opening, but has disappeared by P21. The developmental loss of correlated activity was similar for dark-reared mice. Hence, the effects of dark rearing on correlated activity vary from species to species, possibly because they have different reliances on vision at birth. Newborn mice are not dependent on vision, whereas newly hatched turtles must immediately rely on visual cues for survival (for example marine turtles must run to the sea as soon as they hatch to escape predators and start their long migratory journey). This suggests that the onset of visual experience may be of much more importance in turtle than in mouse retina. Nevertheless, this does not exclude the possibility that a developmental switch in $\mathrm{GABA}_{\mathrm{A}}$ activity is as necessary in mammals as in reptiles to induce the disappearance of correlated SBA. Many more studies manipulating GABAergic systems and light conditions are re-
B

C
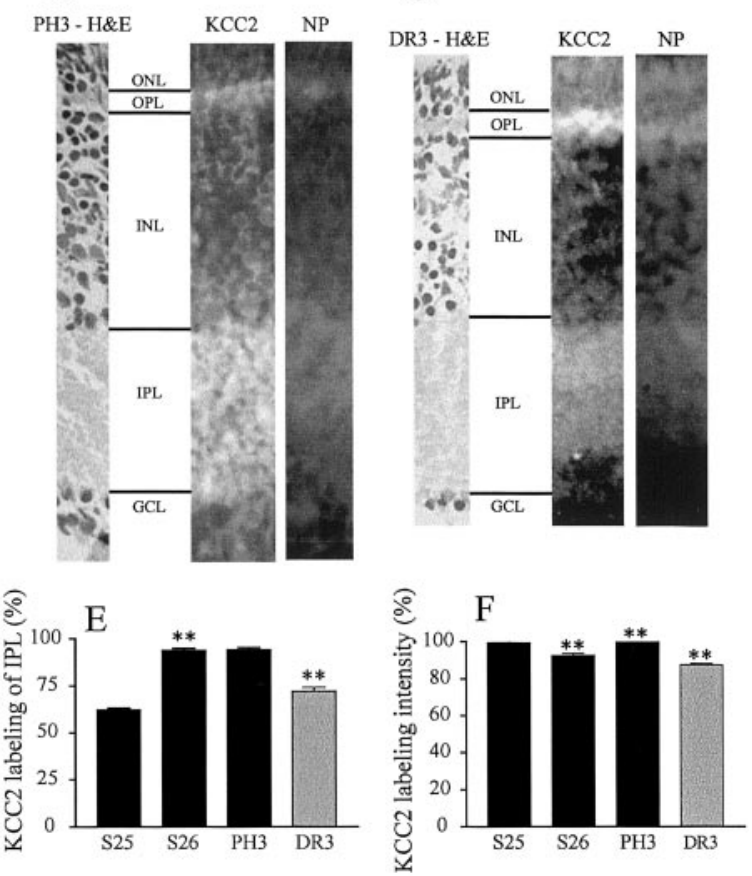

Figure 8. The neural cotransporter KCC2 is upregulated by a light-controlled mechanism during turtle retinal development. $A$, Left panel, Light micrograph of a vertical section through the central retina at S25. Cells and processes are revealed with the H\&E

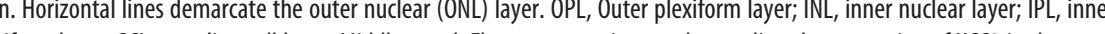

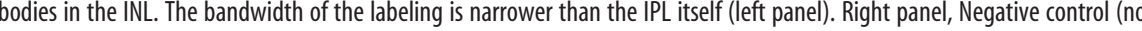
作 Developmental changes in KCC2 labeling intensity. The intensity decreases from S25 to S26, but then peaks at PH3, while it reaches its minimum at DR3. ${ }^{* *} p<0.001 ;{ }^{*} p<0.01$ (N-K post test, each column is compared with the preceding one).

quired to reach a fundamental understanding of these fascinating questions.

\section{References}

Bansal A, Singer JH, Hwang BJ, Xu W, Beaudet A, Feller MB (2000) Mice lacking specific nicotinic acetylcholine receptor subunits exhibit dramatically altered spontaneous activity patterns and reveal a limited role for retinal waves in forming on and off circuits in the inner retina. J Neurosci 20:7672-7681.

Ben-Ari Y (2002) Excitatory actions of GABA during development: the nature of the nurture. Nat Rev Neurosci 3:728-739.

Cardinali DP, Golombek DA (1998) The rhythmic GABAergic system. Neurochem Res 23:607-614.

Catsicas M, Bonness V, Becker D, Mobbs P (1998) Spontaneous $\mathrm{Ca}^{2+}$ transients and their transmission in the developing chick retina. Curr Biol 8:283-286.

Crair MC (1999) Neuronal activity during development: permissive or instructive? Curr Opin Neurobiol 9:88-93.

De Jeu M, Pennartz C (2002) Circadian modulation of GABA function in the rat suprachiasmatic nucleus: excitatory effects during the night phase. J Neurophysiol 87:834-844.

Delpire E (2000) Cation-chloride cotransporters in neuronal communication. News Physiol Sci 15:309-312.

Demas J, Eglen SJ, Wong ROL (2003) Developmental loss of synchronous spontaneous activity in the mouse retina is independent of visual experience. J Neurosci 23:2851-2860.

Eglen SJ (1999) The role of retinal waves and synaptic normalization in retinogeniculate development. Philos Trans R Soc Lond B Biol Sci 354:497-506. 
Ehrlich I, Lohrke S, Friauf E (1999) Shift from depolarizing to hyperpolarizing glycine action in rat auditory neurones is due to age-dependent $\mathrm{Cl}-$ regulation. J Physiol (Lond) 520:121-137.

Feller MB, Wellis DP, Stellwagen D, Werblin FS, Shatz CJ (1996) Requirement for cholinergic synaptic transmission in the propagation of spontaneous retinal waves. Science 272:1182-1187.

Fischer KF, Lukasiewicz PD, Wong ROL (1998) Age-dependent and cell class-specific modulation of retinal ganglion cell bursting activity by GABA. J Neurosci 18:3767-3778.

Ganguly K, Schinder AF, Wong ST, Poo M (2001) GABA itself promotes the developmental switch of neuronal GABAergic responses from excitation to inhibition. Cell 18:521-532.

Grzywacz NM, Sernagor E (2000) Spontaneous activity in developing turtle retinal ganglion cells: statistical analysis. Vis Neurosci 17:229-241.

Jaliffa CO, Saenz D, Resnik E, Keller Sarmiento MI, Rosenstein RE (2001) Circadian activity of the GABAergic system in the golden hamster retina. Brain Res 912:195-202.

Kandler K, Friauf E (1995) Development of glycinergic and glutamatergic synaptic transmission in the auditory brainstem of perinatal rats. J Neurosci 15:6890-6904.

Katz LC, Shatz CJ (1996) Synaptic activity and the construction of cortical circuits. Science 274:1133-1138.

Leinekugel X, Khalilov I, McLean H, Caillard O, Gaiarsa JL, Ben-Ari Y, Khazipov R (1999) GABA is the principal fast-acting excitatory transmitter in the neonatal brain. Adv Neurol 79:189-201.

Maffei L, Galli-Resta L (1990) Correlation in the discharges of neighboring rat retinal ganglion cells during prenatal life. Proc Natl Acad Sci USA 87:2861-2864.

Meister M, Wong ROL, Baylor DA, Shatz CJ (1991) Synchronous bursts of action potentials in ganglion cells of the developing mammalian retina. Science 252:939-943.

Mody I (2001) Distinguishing between $\mathrm{GABA}_{\mathrm{A}}$ receptors responsible for tonic and phasic conductances. Neurochem Res 26:907-913.

Nguyen LT, Grzywacz NM (2000) Colocalization of choline acetyltransferase and gamma-aminobutyric acid in the developing and adult turtle retinas. J Comp Neurol 420:527-538.

O’Donovan MJ (1999) The origin of spontaneous activity in developing networks of the vertebrate nervous system. Curr Opin Neurobiol 9:94-104.

O'Donovan MJ, Ho S, Sholomenko G, Yee W (1993) Real-time imaging of neurons retrogradely and anterogradely labelled with calcium-sensitive dyes. J Neurosci Methods 46:91-106.

Payne JA, Stevenson TJ, Donaldson LF (1996) Molecular characterization of a putative $\mathrm{K}-\mathrm{Cl}$ cotransporter in rat brain. A neuronal-specific isoform. J Biol Chem 271:16245-16252.

Penn AA, Shatz CJ (1999) Brain waves and brain wiring: the role of endogenous and sensory-driven neural activity in development. Pediatric Res 45:447-458.

Penn AA, Riquelme PA, Feller MB, Shatz CJ (1998) Competition in retinogeniculate patterning driven by spontaneous activity. Science 279:2108-2112.

Rivera C, Voipio J, Payne JA, Ruusuvuori E, Lahtinen H, Lamsa K, Pirvola U, Saarma M, Kaila K (1999) The $\mathrm{K}^{+} / \mathrm{Cl}^{-}$co-transporter KCC2 renders GABA hyperpolarizing during neuronal maturation. Nature 397:251-255

Roberts JE (1995) Visible light induced changes in the immune response through an eye-brain mechanism (photoneuroimmunology). J Photochem Photobiol B 29:3-15.

Rossi FM, Pizzorusso T, Porciatti V, Marubio LM, Maffei L, Changeux JP (2001) Requirement of the nicotinic acetylcholine receptor beta 2 subunit for the anatomical and functional development of the visual system. Proc Natl Acad Sci USA 98:6453-6458.

Sernagor E (2002) Neurotransmitter control of wave dynamics in the developing retina. Fed Eur Neurosci Soc Abstr 130-3 (on-line).
Sernagor E, Eglen SJ (2001) Developmental changes in retinal waves dynamics. Program No. 8.1.2001 Abstract Viewer/Itinerary Planner. Washington, DC: Society for Neuroscience.

Sernagor E, Grzywacz NM (1995) Emergence of complex receptive field properties of ganglion cells in the developing turtle retina. J Neurophysiol 73:1355-1364.

Sernagor E, Grzywacz NM (1996) Influence of spontaneous activity and visual experience on developing retinal receptive-fields. Curr Biol 6:1503-1508.

Sernagor E, Grzywacz NM (1999) Spontaneous activity in developing turtle retinal ganglion cells: pharmacological studies. J Neurosci 19:3874-3887.

Sernagor E, Mehta V (2001) The role of early neural activity in the maturation of turtle retinal function. J Anat 199:375-383.

Sernagor E, Eglen SJ, O'Donovan MJ (2000) Differential effects of acetylcholine and glutamate blockade on the spatiotemporal dynamics of retinal waves. J Neurosci 20(RC56):1-6.

Sernagor E, Eglen SJ, Wong ROL (2001) Development of retinal ganglion cell structure and function. Prog Retin Eye Res 20:139-174.

Shatz CJ (1996) Emergence of order in visual system development. Proc Natl Acad Sci USA 93:602-608.

Singer JH, Talley EM, Bayliss DA, Berger AJ (1998) Development of glycinergic synaptic transmission to rat brain stem motoneurons. J Neurophysiol 80:2608-2620.

Stellwagen D, Shatz CJ (2002) An instructive role for retinal waves in the development of retinogeniculate connectivity. Neuron 33:357-367.

Vaney DI (1990) The mosaic of amacrine cells in the mammalian retina. In: Progress in retinal research (Osborne N, Chader J, eds), Vol 9, pp 49-100. Pergamon: Oxford.

Vardi N, Zhang L-L, Payne JA, Sterling P (2000) Evidence that different cation chloride cotransporters in retinal neurons allow opposite responses to GABA. J Neurosci 20:7657-7663.

Vu TQ, Payne JA, Copenhagen DR (2000) Localization and developmental expression patterns of the neuronal $\mathrm{K}-\mathrm{Cl}$ cotransporter (KCC2) in the rat retina. J Neurosci 20:1414-1423.

Williams JR, Sharp JW, Kumari VG, Wilson M, Payne JA (1999) The neuron-specific $\mathrm{K}-\mathrm{Cl}$ cotransporter, $\mathrm{KCC} 2$. Antibody development and initial characterization of the protein. J Biol Chem 274:12656-12664.

Wong ROL (1999) Retinal waves and visual system development. Annu Rev Neurosci 22:29-47.

Wong WT, Wong ROL (2000) Rapid dendritic movements during synapse formation and rearrangement. Curr Opin Neurobiol 10:118-124.

Wong ROL, Meister M, Shatz CJ (1993) Transient period of correlated bursting activity during development of the mammalian retina. Neuron 11:923-938.

Wong ROL, Chernjavsky A, Smith SJ, Shatz CJ (1995) Early functional neural networks in the developing retina. Nature 374:716-718.

Wong WT, Sanes JR, Wong ROL (1998) Developmentally regulated spontaneous activity in the embryonic chick retina. J Neurosci 18:8839-8852.

Yntema CL (1968) A series of stages in the embryonic development of Chelydra serpentina. J Morphol 125:219-251.

Zhou ZJ (1998) Direct participation of starburst amacrine cells in spontaneous rhythmic activities in the developing mammalian retina. J Neurosci 18:4155-4165.

Zhou ZJ (2001) A critical role of the strychnine-sensitive glycinergic system in spontaneous retinal waves of the developing rabbit. J Neurosci 21:5158-5168.

Zhou ZJ, Zhao D (2000) Coordinated transitions in neurotransmitter systems for the initiation and propagation of spontaneous retinal waves. J Neurosci 20:6570-6577.

Zhou ZJ, Lee S, Syed MM (2002) Multistage and multidimensional retinal waves suggest diverse developmental roles. Program No. 236.1. $2002 \mathrm{Ab}-$ stract Viewer/Itinerary Planner. Washington, DC: Society for Neuroscience. 